\title{
M edicamentos de alto custo para doenças raras no Brasil: o exemplo das doenças lisossômicas
}

\author{
High cost drugs for rare diseases in Brazil: \\ the case of lysosomal storage disorders
}

M ônica Vinhas de Souza ${ }^{1}$

Bárbara Corrêa Krug ${ }^{1}$

Paulo Dornelles Picon ${ }^{2}$

Ida Vanessa D oederlein Schwartz ${ }^{3}$

\footnotetext{
${ }^{1}$ Programa de PósGraduação em Ciências M édicas, Faculdadede M edicina, U niversidade Federal do Rio Grandedo Sul. Praça Dr. M auricio Cardoso, 115/302. 90570010 Porto Alegre RS. vsmonica@uol.com.br. ${ }^{2}$ Serviço de M edicina Interna, Hospital deClínicas dePorto Alegre.

${ }^{3}$ Departamento deGenética, UniversidadeFederal do Rio Grandedo Sul.
}

Abstract This paper approachesin a critical way aspects of Brazilian public policies for drugs, emphasizing those classified as high cost and for rare diseases. Thelysosomal storage diseases wastaken as an example because of their rarity and the international trend for the development of new drugs for their treatment, all at high costs. Three lysosomal storage diseases were approached: Gaucher disease, Fabry disease and mucopolysaccharidosis type I. Gaucher disease has its treatment drug licensed in Brazil and guidelines for its use are established through a dinical protocol by the M inistry of $\mathrm{H}$ ealth. The others have their drug treatments registered in Brazil; however, no treatment guidelines for them have been developed by the government. The objective of the paper was to foster the discussion on the role of health technology assessment for high-cost drugs for rare diseases in Brazil, emphasizing the need for establishing health policies with legitimacy towards these diseases. Despite the difficulties in establishing a health policy for each rare disease, it is possible to create rational models to deal with this growing challenge.

Key words Rare diseases, Lysosomal storage diseases, High-cost drugs, H ealth policies
Resumo Esteartigo aborda, de forma crítica, aspectos das políticas públicas brasileiras para medicamentos, com ênfasenos de alto custo dirigidosàs doenças raras. As doenças lisossômi cas foram utilizadas como exemplo pela sua raridadeepela tendência mundial para o desenvolvimento de novos fármacos para seu tratamento. Três doenças foram abordadas: doença de Gaucher, doença de Fabry e mucopolissacaridose tipo I. Embora todas tenham medicamentos registrados no Brasil, a doença de Gaucher é a única com protocolo clínico e diretrizes de tratamento balizadas pelo M inistério da Saúde. Os autores almejam, com este artigo, fomentar a discussão sobre o papel da avaliação de tecnologias em saúde para o tratamento das doenças raras no Brasil, enfatizando a necessidade de políticas legitimadas dirigidas especialmentea elas. A despeito das dificuldades de se estabelecer uma política de saúde específica para cada doença rara, é possível o estabelecimento de modelos racionais para lidar com esse crescente desafio.

Palavras-chave Doenças raras, Doenças lisossômicas, M edicamentos de alto custo, Políticas de saúde 
Introdução

Os erros inatos do metabolismo (EIM) constituem-se em um grupo heterogêneo de doenças genéticas raras, caracterizadas pela presença de mutações patogênicas em genes que codificam enzimas envolvidas em alguma rota do metabolismo. No caso das doenças lisossômicas, as enzimas que apresentam atividade deficiente são aquelas envolvidas no catabolismo intralisossomal de macromoléculas. São descritas aproximadamente ciquenta doenças lisossômicas ${ }^{1}$, com incidência conjunta estimada em 1:7.000 recémnascidos vivos ${ }^{2,3}$. Em um estudo realizado no Laboratório de Referência para Erros Inatos do M etabolismo do Hospital de Clínicas de Porto Alegre/RS (LREIM - HCPA), centro de referência para o diagnóstico EIM no Brasil, as doenças lisossômicas corresponderam a 59,8\% dos EIM diagnosticados ${ }^{4}$. Algumas dessas doenças, como por exemplo a doença de Gaucher, são tratadas por terapia de reposição enzimática (TRE), uma modalidade de tratamento deal to custo queconsiste na aplicação periódica de uma enzima artificial, semelhanteàquela queo paciente não produz em quantidadesuficientee cuja deficiênciaé a responsável pelo quadro clínico da doença.

O Sistema Único de Saúde (SUS) não possui política de assistência farmacêutica específica para doenças raras, e a própria construção dessa política esbarra em questões bióeticas que envolvem temas como equidade, recursos escassos e reserva do possível. Mesmo assim, a demanda por TRE é cada vez maior, sendo às vezes balizada por ordens judiciais que entram em conflito com a Política Nacional de M edicamentos ${ }^{5}$ ecom a medicina baseada em evidências (MBE). Há de se considerar, também, o lobby da indústria farmacêutica para a entrada de novos fármacos no país.

Pretende-se, com este artigo, iniciar-se uma reflexão multidisciplinar sobre a necessidade de model os para avaliação de tecnologia em saúde (ATS) e de políticas envolvendo doenças raras e tratamentos inovadores no SUS. A doença de Gaucher, a mucopolissacaridose I e a doença de Fabry foram escolhidas para ilustrar esta discussão porque reúnem aspectos modelares para a análise dos determinantes envolvidos na aquisição de biotecnologias ante recursos escassos.

\section{Doença de Gaucher}

A doença de Gaucher (deficiência da $\beta$-glicosidase ácida) foi a primeira doença lisossômica a ser tratada por meio deTRE. São descritos três tipos principais desta doençá: (1) tipo I - é o mais comum, sendo caracterizado pelo quadro visceral (hepatoesplenomegalia), envolvimento ósseo e hematológico (anemia, plaquetopenia), e ausência de comprometimento neurológico; (2) tipo II - as manifestações clínicas são muito precoces e há comprometimento neurológico importante e grave; e (3) tipo III - é semelhante ao tipo II, porém menos precoce e menos grave. Os pacientes com doença de Gaucher tipo II, manifestação mais grave dela, têm uma expectativa de vida muito curta e morrem ainda na primeira infância, geralmente antes dos três anos de idade. 0 comprometimento neurológico inclui regressão neurológica e convulsões.

Desde o início da década de 1990, a doença de Gaucher tipo I sintomática eas manifestações não neurológicas da doença de Gaucher tipo III têm sido tratadas com sucesso através da TRE com imiglucerase, uma enzima recombinante muito semelhante à $\beta$-glicosidase ácida, sem similares no mercado. Não existem evidências de eficácia desta forma de terapia na doença de Gaucher tipo II ${ }^{7}$. Desde 1998, a imiglucerase faz parte da lista dos excepcionais do M inistério da Saúde do Brasil (MS) (Tabela 1). A imiglucerase éum medicamento dealto custo cujo fabricanteéúnico (Genzyme Corporation). Em 2007, aproximadamente quinhentos pacientes com doença de Gaucher foram tratados no Brasil, sendo comprados 177.280 frascos a um custo de mais de $R \$ 250$ milhões$^{8}$. Até2002, não existiam diretrizes nacionais para 0 uso de imiglucerase apoiadas pelo MS. Naquele ano foi publicado, pelo próprio MS, na forma de portaria, o Protocolo Clínico e Diretrizes Terapêuticas para doença de Gaucher (PCDT-Gau(her) 7 . O PCDT-Gaucher foi construído com auxílio de duas autoridades internacionais (Dr. Ernest Beutler, EUA, e Dr. Ari Zimran, Israel) e discutido em consulta pública nacional. Este protocolo criou o conceito de "menor doseclinicamente eficaz" de imiglucerase, o que recomenda que seja buscado sempre que possível.

Outras estratégias específicas de tratamento estão atualmente sendo testadas para a doença de Gaucher e para as demais doenças lisossômicas (Tabela 2). Algumas delas, inclusive, já foram aprovadas por agências internacionais de regulamentação (Tabela 2). A maioria desses tratamentos é considerada de alto custo mesmo para países desenvolvidos, e estão sendo desenvolvidos para doenças consideradas até então sem tratamento, como as mucopolissacaridoses. No caso das doenças lisossômicas para as quais já existe trata- 
mento (usualmenteTRE), é muito remota a possibilidade de que sejam feitos ensaiosfase III, duplo-cegos, randomizados contra a terapia convencional, uma vez quea "terapia convencional" e a "nova terapia" foram desenvolvidas por empresas concorrentes. Esse problema já vem acontecendo em relação à doença de Gaucher: não foi considerada ética, por exemplo, a realização de um ensaio clínico faselll comparando aTRE com imiglucerase e um medicamento novo (inibidor da síntese de substrato ou miglustate); isso fez com queo miglustate fosse aprovado, pelas agências de regulação de medicamentos americana (Food and DrugAdministration ou FDA) eeuropeia (European M edicinesAgency ou EM EA), para uso somente em um pequeno subgrupo de pacientes com doença de Gaucher (aqueles que não toleravam a TRE), pois estes foram os únicos pacientes que puderam ser incluídos, por motivos éticos, nos ensaios clínicos do novo fármaco. Connock et al. ${ }^{9}$ consideram este princípio ético equivocado; segundo estes autores, a posição de monopólio da TRE não deve ser utilizada como desculpa para impedir a realização de pesquisas fundamentais sobre intervenções alternativas, e que talvez sejam até mais custo-efetivas.

\section{Mucopolissacaridosetipo I}

A mucopolissacaridosetipo I (M PSI) éuma doença lisossômica causada pela atividade deficiente da enzima $\alpha$-L-iduronidase (Q uadro 1 ), com incidência mundial estimada em $1 / 100.000$ recémnascidos vivos ${ }^{2,3}$. N ão existem estudos epidemiológicos sobre esta doença no Brasil. Segundo o Registro deM PSI, financiado pela GenzymeCorporation, existem aproximadamente 55 pacientes identificados em nosso país ${ }^{10}$. Este número é provavelmente uma subestimativa, uma vez que a RedeM PS Brasil, iniciativa coordenada por uma das autoras deste artigo (IVD Schwartz), cadastrou, de abril/2004 a outubro/2007, 87 pacientes brasileiros.

A MPS I está associada a três formas clássicas, quediferem entresi com base na presença de comprometimento neurológico, navelocidadede progressão da doença e na gravidade do acome-

Tabela 1. Medicamentos para doenças genéticas incluídos no componente de medicamentos de dispensação excepcional*.

\begin{tabular}{|c|c|c|c|}
\hline Denominação comum brasileira & Doença & $\begin{array}{l}\text { Incidência** } \\
\text { (/100.000 RNs) }\end{array}$ & $\begin{array}{c}\text { Possui } \\
\text { PCDT/M S? }\end{array}$ \\
\hline Acitretina & Ictioses & $1: 300.000$ & Sim a \\
\hline Alfadornase & Fibrose cística & $12,5-50$ & $\operatorname{Sim} b$ \\
\hline Atorvastatina, fluvastatina, lovastatina, & Dislipidemias & & \\
\hline pravastatina e sinvastatina & & 200 & $\operatorname{Sim}{ }^{b}$ \\
\hline Danazol & Angioedema hereditário & Desconhecido & $\operatorname{Sim}^{\mathrm{c}}$ \\
\hline Desferroxamina & Hemoglobinopatias & 700 & Não \\
\hline Fórmula isenta fenilalanina ( $<1$ ano) & Fenilcetonúria & 10 & $\operatorname{Sim}^{b}$ \\
\hline Fórmula isenta fenilalanina ( $>1$ ano) & Fenilcetonúria & 10 & $\operatorname{Sim}{ }^{b}$ \\
\hline Hidroxiureia & H emoglobinopatias & 700 & Sim \\
\hline Imiglucerase & Doença de Gaucher & $1-2$ & $\operatorname{Sim} \mathrm{b}$ \\
\hline Imunoglobulina humana & Imunodeficiências primárias & 10 & Sim d \\
\hline Levotiroxina & Hipotireoidismo congênito & 200 & $\operatorname{Sim} b$ \\
\hline Pancreatina e pancrelipase & Fibrose cística & $12,5-50$ & Sim \\
\hline Penicilamina & Doença de Wilson & $3-4$ & $\operatorname{Sim}{ }^{b}$ \\
\hline Somatropina & Deficiência de hormônio do crescimento & $10-25$ & Sim a \\
\hline Somatropina & Síndrome de Turner & 40 (sexo feminino) & Sim a \\
\hline
\end{tabular}

*Portaria GM no 2.577/06 do Ministério da Saúde ${ }^{30} ;{ }^{*}$ Cogan e Phillips ${ }^{42} ;$ Culotta e Gitlin ${ }^{43}$; First e H urst ${ }^{44}$. Doença órfã: $<200.000$ casos/ano ou 75/100.000 habitantes ${ }^{27}$, prevalência $<50 / 100.000$ habitantes $^{28}$.

a PCDT/M S45; b PCDT/M S46; c Em consulta pública (CP no 3, de 13/06/2008) 47; d PCDT/M S48.

0 pamidronato dissódico consta da lista de medicamentos excepcionais, mas não para o tratamento das osteogêneses imperfeitas (O I). 0 tratamento das OI é regulado pela Portaria GM n².305/200149. RN s: recém-nascidos vivos;

PCDT/M S: Protocolos Clínicos e Diretrizes Terapêuticas do M inistério da Saúde. 
timento dos órgãos-alvo (sistema nervoso central, ossos, articulações, vias aéreas superiores e inferiores, coração e córnea, principalmente) ${ }^{11}$. $\mathrm{Na}$ forma grave (síndrome de Hurler), os paci- entes costumam ser diagnosticados até os dois anos de idade, apresentar atraso de desenvolvimento aparente entre 14 e 24 meses e estatura máxima de $110 \mathrm{~cm}$; a história clínica édominada

\begin{tabular}{|c|c|c|c|c|c|c|}
\hline $\begin{array}{l}\text { Doença- } \\
\text { alvo }\end{array}$ & Nome do fármaco & $\begin{array}{l}\text { M ecanismo } \\
\text { de ação }\end{array}$ & $\begin{array}{c}\text { Fase de } \\
\text { desenvolvimento }\end{array}$ & $\begin{array}{l}\text { Registro } \\
\text { Anvisa? }\end{array}$ & $\begin{array}{l}\text { Aprovada pelo } \\
\text { FDA/EMEA? }\end{array}$ & $\begin{array}{l}\text { Custo por paciente } \\
\text { (média anual para } \\
\text { medicamentos aprovados } \\
\text { no país de origem) }\end{array}$ \\
\hline Fabry & Betagalsidase & TRE & Pós-comercialização & SIM & SIM (ambos) & Inglaterra: US\$215 mil \\
\hline Fabry & Alfagalsidase & TRE & Pós-comercialização & NÃ $O^{* *}$ & SIM (EMEA) & Inglaterra: US\$ $215 \mathrm{mil}$ \\
\hline Fabry & AT 1001 & Chaperona & Fase I/II & NÃO & NÃO & - \\
\hline Gaucher & $\begin{array}{l}\text { Velaglucerase alfa } \\
\text { (glicocerebrosidase } \\
\text { produzida em } \\
\text { células humanas) }\end{array}$ & TRE & Fase III & $\begin{array}{l}\text { N ÃO } \\
\text { N } \tilde{O} O\end{array}$ & $\begin{array}{l}\text { NÃO } \\
\text { NÃO }\end{array}$ & - \\
\hline Gaucher & $\begin{array}{l}\text { Glicocerebrosidase } \\
\text { produzida em } \\
\text { células vegetais }\end{array}$ & TRE & $\begin{array}{l}\text { Inicial (fase I } \\
\text { finalizada em } \\
\text { voluntários sadios) }\end{array}$ & SIM & $\begin{array}{l}\text { SIM (ambos), } \\
\text { para pacientes que } \\
\text { não toleram TRE } \\
\text { convencional }\end{array}$ & $?$ \\
\hline Gaucher & M iglustate & ISS & $\begin{array}{l}\text { Ensaio clínico aberto } \\
\text { em pacientes com } \\
\text { doença tipo I } \\
\text { estabilizada e que } \\
\text { estavam em TRE com } \\
\text { enzima convencional }\end{array}$ & NÃO & NÃO & - \\
\hline Gaucher & Genz-112638 & ISS & Fase $1 / I I$ & NÃO & NÃO & - \\
\hline Gaucher & AT2101 & Chaperona & Inicial & NÃO & NÃO & - \\
\hline MPS I & Laronidase & TRE & Pós-comercialização & SIM & SIM (ambos) & Inglaterra: US\$ 260 mil \\
\hline MPS II & Idursulfase & TRE & Pós-comercialização & SIM & SIM (ambos) & $?$ \\
\hline MPS VI & Galsulfase & TRE & Pós-comercialização & SIM & SIM (ambos) & US\$ $260 \mathrm{mil}$ \\
\hline $\begin{array}{l}\text { Niemann- } \\
\text { Pick B }\end{array}$ & $\begin{array}{l}\text { Esfingomielinase } \\
\text { recombinante }\end{array}$ & TRE & Inicial & NÃO & NÃO & -1 \\
\hline $\begin{array}{l}\text { Niemann- } \\
\text { Pick C }\end{array}$ & M iglustate & ISS & $\begin{array}{l}\text { Ensaio clínico } \\
\text { randomizado }\end{array}$ & NÃO & NÃO & - \\
\hline Pompe & $\begin{array}{l}\text { Alglucosidase } \\
\text { alfa( } \alpha \text {-glicosidase } \\
\text { ácida produzida em } \\
\text { células } \mathrm{CHO} \text { ) }\end{array}$ & TRE & $\begin{array}{l}\text { Pós-comercialização } \\
\text { (aprovada sem } \\
\text { realização de estudo } \\
\text { controlado por } \\
\text { placebo) }\end{array}$ & NÃO & SIM (ambos) & $\begin{array}{c}\text { Estados Unidos: } \\
\text { US } \$ 200 \text { mil-US\$ } 300 \text { mil }\end{array}$ \\
\hline Pompe & $\begin{array}{l}\alpha \text {-glicosidase ácida } \\
\text { recombinante } \\
\text { produzida a partir } \\
\text { do leite de coelhos } \\
\text { transgênicos }\end{array}$ & TRE & $\begin{array}{l}\text { Fase I/II } \\
\text { Fase I/II } \\
\text { Fase I }\end{array}$ & NÃO & NÃO & - \\
\hline $\begin{array}{l}\text { Tay-Sachs } \\
\text { (tardia) }\end{array}$ & M iglustate & ISS & & NÃO & NÃO & - \\
\hline $\begin{array}{l}\text { Tay-Sachs } \\
\text { (tardia) }\end{array}$ & Pirimetamina & Chaperona & & NÃO & NÃO & - \\
\hline
\end{tabular}

*Foram incluídos somente tratamentos que estão na fase de estudos clínicos ou quejá foram aprovados por agências internacionais. Não foram incluídos protocolos de terapia gênica ou detransplante de células hematopoiéticas. Não existem Protocolos Clínicos e Diretrizes Terapêuticas do M inistério da Saúde para tais medicamentos.

${ }^{* *}$ A alfagalsidase foi licenciada pela Anvisa após a finalização deste artigo, em outubro de 2009.

M PS: mucopolissacaridose; TRE: terapia de reposição enzimática; ISS: inibição da síntese do substrato; ?: informação não encontrada naliteratura. 


\begin{tabular}{|c|c|c|c|}
\hline \multicolumn{4}{|c|}{ Quadro 1. Classificação das mucopolissacaridoses ${ }^{11}$. } \\
\hline $\begin{array}{l}\text { Tipo de } \\
\text { MPS }\end{array}$ & Enzima com atividade deficiente & $\begin{array}{l}\text { Glicosaminoglicano(s) } \\
\text { não degradado(s) }\end{array}$ & Epônimo \\
\hline I & $\alpha$-L-iduronidase & DS, HS & Hurler Hurler-Scheie Scheie \\
\hline II & $\begin{array}{l}\text { iduronato-sulfatase } \\
\text { sulfamidase (heparan N-sulfatase) }\end{array}$ & DS, HS & Hunter \\
\hline IIIA & $\alpha-\mathrm{N}$-acetilglicosaminidase & HS & Sanfilippo A \\
\hline IIIB & acetil-Coa: $\alpha$-glicosaminida & HS & Sanfilippo B \\
\hline IIIC & acetiltransferase & HS & Sanfilippo C \\
\hline IIID & $\mathrm{N}$-acetilglicosamina 6-sulfatase & HS & Sanfilippo D \\
\hline IVA & $\begin{array}{l}\mathrm{N} \text {-acetil-galactosamina 6-sulfatase } \\
\text { (galactose-6-sulfatase) }\end{array}$ & QS, CS6 & Morquio A \\
\hline IVB & $\beta$-galactosidase & QS & Morquio B \\
\hline VI & $\begin{array}{l}\mathrm{N} \text {-acetilgalactosamina 4- sulfatase } \\
\text { (arilsulfatase B) }\end{array}$ & DS, CS4 & M aroteaux-Lamy \\
\hline VII & $\beta$-glicuronidase & DS, HS, CS4, CS6 & Sly \\
\hline IX & hialuronidase & hialuronan & Natowicz \\
\hline
\end{tabular}

DS: dermatan sulfato; HS: heparan sulfato; QS: queratan sulfato; CS4: condroitin 4-sulfato; CS6: condroitin 6-sulfato.

por problemas respiratórios, e o óbito ocorre geralmente durante a primeira década de vida, por insuficiência cardíaca ou respiratória. $\mathrm{Na}$ forma intermediária (síndrome de HurlerScheie), os pacientes costumam apresentar evidência clínica da doença entre três e oito anos de idade, sendo que a baixa estatura final é importante; a maioria dos pacientes apresenta inteligência normal, ea sobrevivência atéa idade adulta é comum. $\mathrm{Na}$ forma atenuada (síndrome de Scheie), a sintomatologia dos pacientes costuma iniciar entre cinco e quinze anos de idade, sendo que o curso clínico é dominado por problemas ortopédicos; a altura final é normal ou quase normal, assim como o tempo de vida, o qual pode se mostrar reduzido pela doença cardíaca.

Não existe tratamento curativo para a M PSI. As opções terapêuticas disponíveis incluem intervenções realizadas no nível do fenótipo clínico (tais como cirurgias para correção de hérnias) ou no nível da proteína mutante (transplante de células hematopoiéticas e TRE com laronidase). A laronidase, $\alpha$-L-iduronidase recombinante, é produzida pela Biomarin Pharmaceutical Inc. e pela Genzyme Corporation, EUA. Existe, até 0 momento, apenas um ensaio clínico de fase III, duplo-cego, randomizado contra placebo, publicado a seu respeito na literatura, o qual incluiu 45 pacientes $^{12}$. Em 2003, este produto foi aprovado pelo FDA para o tratamento das formas graves, intermediárias e atenuadas, com sintomas graves ou moderados da MPS I. Em junho do mesmo ano, foi aprovado também pela EMEA. Em contrapartida, em 2005, o Canadian Expert Drug Advisory Committee (CEDAC), administrado pelo Canadian Coordinating Office on $\mathrm{H}$ ealth Technology Assessment, não recomendou o financiamento da laronidase no Canadá, pois os estudos não teriam demonstrado benefício em desfechos clínicos e haveria o risco de desenvolvimento de eventos adversos graves; além disso, o tratamento não foi considerado custo- efetivo ${ }^{13}$.

A laronidase é um medicamento de alto custo que recebeu registro na Agência $\mathrm{N}$ acional de Vigilância Sanitária (Anvisa) em 2005, mas que não se encontra incluído na lista de medicamen- 
tos excepcionais do MS. Estima-se que aproximadamente quarenta pacientes brasileiros estejam sendo atualmente tratados com laronida$\mathrm{se}^{14}$, provavelmente via programa de acesso expandido (subsidiado pela indústria), ou como uso comercial (financiado diretamente pelos governos estaduais ou União).

Não existem estudos sobre avaliação econômica da TRE para M PS I publicados na literatura. Connock et al. ${ }^{9}$ tentaram, sem sucesso, desenvolver um modelo de custo-efetividade para a laronidase, tendo atribuído o insucesso à falta de dados sobre a história natural da MPS I e sobreo efeito daTRE em desfechos centrais como mortalidade.

\section{Doença de Fabry}

A doença de Fabry étambém uma doença lisossômica, de herança ligada ao X, na qual os pacientes do sexo masculino apresentam atividade deficiente da enzima lisossômica $\alpha$-galactosidase A ( $\alpha G A L-A)$. As heterozigotas podem ser assintomáticas ou desenvolver a doença, possivelmentedevido à inativação randômica deum cromossomo $X^{15}$. A incidência estimada da doença de Fabry é de 1 em 40.000 a 100.000 indivíduos do sexo masculino ${ }^{15,16}$.

Ao contrário da maioria das doenças lisossômicas, a doença de Fabry éprimariamenteuma doença de adultos ${ }^{17}$. Na forma clássica da doença (a mais frequente), os pacientes iniciam com acroparestesias entre cinco e dez anos de idade. $\mathrm{Na}$ maioria dos casos, na idade adulta, os pacientes desenvolvem doenças renal e cardíaca graves, sendo a disfunção renal progressiva a principal causa de óbito ${ }^{17}$.

A betagalsidase é uma forma recombinante da enzima humana $\alpha G A L-A$, produzida pela Genzyme Corporation a partir de células $\mathrm{CHO}$ (Tabela 2), e que foi aprovada, em 2003, pelo FDA e pela EM EA, para o tratamento da doença de Fabry. Essa aprovação foi baseada em um único ensaio clínico fase III, duplo-cego, randomizado contra placebo, e que incluiu 58 pacientes ${ }^{18}$. Em 2007, mais um ensaio clínico randomizado com betagalsidase foi publicado, desta vez incluindo 82 pacientes, tendo sido o primeiro focado em desfechos clínicos (primeiro evento cardíaco, renal ou neurológico) ${ }^{19}$. Este fármaco já possui registro na Anvisa, éde al to custo e não faz parte da lista de medicamentos excepcionais.

A alfagalsidase, produzida a partir de células humanas pela Shire HGT, EUA, é a outra opção para o tratamento da doença de Fabry. Foi aprovada para uso na União Europeia, com base no estudo de Schiffmann et al. ${ }^{20}$, o qual incluiu 26 pacientes, mas não pelo FDA, e também não possui registro na Anvisa.

Embora a dose recomendada de betagal sidase $(1,0 \mathrm{mg} / \mathrm{kg})$ seja cinco vezes superior à dose recomendada dealfagalsidase $(0,2 \mathrm{mg} / \mathrm{kg}), 0$ custo dos tratamentos é semelhante 9 . Recentemente foi publicado um ensaio clínico randomizado quecomparou ambas as enzimas em doses iguais (0,2 mg/kg, quinzenalmente); neste estudo, não houve diferença entre ambos os grupos, quer para parâmetros de resposta às medicações, quer para falhas no tratamento ${ }^{21}$. Existem outras evidências que sugerem que a alfa e a betagal sidase sejam proteínas funcionalmenteindistinguíveis9.

O único estudo sobre custo-efetividade da TRE na doença de Fabry é o de Connock et al. ${ }^{9}$, cujos resultados apontam para o fato de que esse tratamento não é custo-efetivo.

O LREIM-HCPA diagnosticou, de 1982 a 2007, em torno de cinquenta pacientes brasileiros do sexo masculino com doença de Fabry (o total de pacientes brasilei ros é certamente superior a este número, entre outros motivos porque - levantamento deste laboratório não incluiu indivíduos do sexo feminino). Estima-se que pelo menos quarenta pacientes estejam recebendo TRE no Brasil (a maioria com betagalsidase), provavelmente via programa de acesso expandido ou como uso comercial.

Nesse ponto, cabe um comentário: uma vez que é real e premente a perspectiva de que os tratamentos que constam na Tabela 2 sejam incorporados no SUS nos próximos dez anos, talvez fosse importante a adoção pelo governo brasileiro de medidas para o desenvolvimento de mecanismos que propiciassem a realização de ensaios clínicos nessa área, coordenados e subsidiados pelo governo. Como certamente é o governo que vai arcar com os custos de tais tratamentos (pois todos serão necessariamente dealto custo), os ensaios clínicos poderiam ser desenhados conforme a sua demanda, comparando, por exemplo, as diversas formas de tratamento, inclusive combinados. A adoção desse tipo de estratégia depende da resolução do seguinte conflito: a responsabilidade pela criação de evidências é, prioritariamente, da indústria farmacêutica, interessada na comercialização dos seus medicamentos, ou do Estado, o seu financiador?

A experiência do Canadá deve ser ressaltada: - CEDAC não recomendou o financiamento, pelo governo canadense, da TRE para pacientes com 
doença de Fabry (com quaisquer dos dois produtos disponíveis: alfa ou betagalsidase), devido à falta de evidência consistente sobre a sua eficá$\mathrm{cia}^{22,23}$. Em 2006, entretanto, o governo canadenseresolveu participar deum ensaio clínico envolvendo pacientes com doença de Fabry e incluindo ambos os fármacos, a fim de serem obtidas evidências adicionais sobre a sua eficácia e custoefetividade ${ }^{24}$.

\section{M edicamentosórfãos}

Um tópico especial na política de fiscalização e regulação de medicamentos em todo 0 mundo vem a ser os chamados "medicamentos órfãos". Este termo foi usado pela primeira vez em 1968 para descrever medicamentos potencialmente úteis, não disponíveis no mercado; sua exploração não era considerada lucrativa por motivos como dificuldade de produção ou, ainda, por serem destinados ao tratamento de doenças raras $^{25}$. Para a atribuição do status de medicamento órfão, dois conceitos são utilizados conjuntamente: o epidemiológico ( prevalência ou incidência da doença numa população) e o econômico (presunção denão rentabilidade do medicamento destinado à terapêutica da doença em questão). 0 princípio quenorteia a maioria das agências de saúde em todo o mundo é o de que a "raridade" das chamadas "doenças órfãs" não produz lucratividade para as empresas farmacêuticas, desestimulando-as a investir na pesquisa e no desenvolvimento de medicamentos para essas enfermidades, e colocando os portadores dessas doenças em uma situação de desvantagem e fragilidade. Este aspecto, por sua vez, determinaria a necessidade da existência de políticas governamentais especiais para esse grupo de doenças e medicamentos ${ }^{26}$.

O FDA criou, em 1982, um setor específico para os "medicamentos órfãos"; a isto se seguiu o O rphan DrugAct, aprovado em 1983 pelo Congresso Americano. Este não somente caracteriza o medicamento órfão como aquele direcionado para uma doença que atinja menos de 200.000 casos/ano nos EUA (cerca de 75/100.000 habitantes) como cria, para os medicamentos assim classificados, linhas especiais de financiamento governamental, impostos diferenciados e permite ainda que eles passem por protocolos especiais de investigação e aprovação mais rápidos que os usuais ${ }^{27}$. O sucesso do Orphan Drug Act pode ser exemplificado pelo fato de que, entre $1972 \mathrm{e}$ 1982, o FDA aprovou dez tratamentos para do- enças raras, número que aumentou, entre $1982 \mathrm{e}$ 2006, para 282; os pacientes mais beneficiados foram os portadores de formas raras de câncer $e$ de doenças metabólicas (como os EIM) ${ }^{26}$.

A EMEA possui também, desde 1999, uma política bem definida em relação aos chamados medicamentos órfãos. Os critérios epidemiológicos são claros: Os medicamentos órfãos são destinados à prevenção, diagnóstico ou tratamento de condições muito graves, ou que signifiquem um risco para a vida, eque sejam extremamenteraras, com uma prevalência dentro da União Européia igual ou inferior a 50/100.000 habitantes ${ }^{28}$. A caracterização de um medicamento como "órfão" junto ao EM EA possibilita queo desenvolvimento deste conte com aconselhamento científico da agência, obtenção de subsídios variados, redução nos impostos associados e, em última instância, atéuma exclusividade de mercado nosdez anos seguintes à sua aprovação de venda nos países da União Europeia ${ }^{28}$. A agência europeia ressalta em suas diretrizes específicas sobre 0 assunto que, para muitas das chamadas "doenças órfãs", a experiência nos tratamentos específicos é restrita, quer pela inovação do tratamento, quer pela singularidade da doença, o que determina uma limitação na realização de ensaios clínicos comparativos pautados em princípios e métodos já existentes. N esses casos, ocorre muitas vezes a necessidade de utilização ainda mais ampla de modelos in vitro e/ou pré-clinicos para a avaliação desses medicamentos. A EM EA enfatiza a necessidade de que conceitos como o da "plausibilidade científica" e"benefício plausível" não sejam negligenciados, sendo sempre desejável a utilização de métodos de avaliação baseados na metodologia científica convencional, e ainda em aspectos inovadores como a farmacogenética ${ }^{28}$.

No entanto, deve ser ressaltado que incentivos para o desenvolvimento de medicamentos órfãos podem, paradoxalmente, estimular 0 monopólio de indústrias farmacêuticas e tornar tais medicamentos altamente lucrativos, o que estaria em desacordo com a definição inicialmente proposta para "medicamento órfão".

No Brasil, a definição empregada pela Anvisa para "medicamentos órfãos" diz que estes são medicamentos "utilizados em doenças raras, cuja dispensação atende a casos específicos". Este conceito é pouco claro e sequer explicita a definição de "doença rara" no contexto do nosso país ${ }^{29}$. Chama a atenção não apenas a definição "sintética" eincompleta empregada pela nossa agência para o termo "medicamentos órfãos", mas tam- 
bém a inexistência deuma política específica para este grupo de fármacos, alguns dos quais se encontram inseridos no componente de medicamentos de dispensação excepcional $(\mathrm{CM} \mathrm{DE})^{30}$. Esses medicamentos são aqueles utilizados para tratamento de agravos inseridos nos seguintes critério ${ }^{30}$ : (1) doença rara ou de baixa prevalência, com indicação de uso de medicamento de alto valor unitário ou que, em caso de uso crônico ou prolongado, seja um tratamento de custo elevado; e (2) doença prevalente, com uso de medicamento de alto custo unitário ou que, em caso de uso crônico ou prolongado, seja um tratamento de custo elevado.

O CM DE surgiu, em 1993, com a denominação de "Programa de Medicamentos Excepcionais", e seu objetivo inicial era o de disponibilizar gratuitamenteà população medicamentos de uso ambulatorial, de alto custo, e que não pudessem ser comprados pelos cidadãos ${ }^{31}$. O CM DE continua sendo um programa que visa à cobertura de medicamentos ambulatoriais de alto custo. $N$ ão existe consenso sobre a definição de medicamentos de alto custo; uma das propostas diz que me dicamentos dealto custo são aqueles cujo valor unitário mensal esteja acima de um salário mínimo, ou medicamentos de uso crônico indicado para doenças muito prevalentes (acima de $1 \%$ da população) cujo custo mensal seja superior a um terço de um salário mínimo ${ }^{32}$. Este programa/componente é cofinanciado pelos governos estadual e federal, e possui uma lista de medicamentos específica, definida na própria portaria de sua criação.

Alguns problemas são evidenciados nesse cenário: (1) apesar de o programa existir desde 1993 e de sofrer modificações subsequentes (inclusão e exclusão de medicamentos), carece ainda de diretrizes claras que estabeleçam seu conceito e os critérios de inclusão/exclusão de medicamentos na lista; e (2) o programa custou, em 2005, aproximadamente $R \$ 1,2$ bilhão (cerca de $1 / 3$ dos investimentos para todos os programas de medicamentos) ${ }^{33}$. Quatorze medicamentos são responsáveis por $60 \%$ deseu custo. Entreeles, incluem-se dois medicamentos utilizados no tratamento de doenças genéticas: imiglucerase (para doença de Gaucher) e a alfadornase (para fibrose cística) ${ }^{33}$. Em resumo, a maior parte desse orçamento destina-se ao tratamento de um pequeno número de pacientes.

A inserção dos medicamentos para doenças raras dentro do CMDE traz, na opinião dos autores, alguns inconvenientes. 0 mais importante deles é que os medicamentos para doenças menos prevalentes ficam sujeitos às mesmas regras de avaliação que os medicamentos para doenças mais prevalentes. A própria Organização M undial da Saúde (OMS) levantou, em 2006, uma discussão sobrea necessidade da criação deum novo tipo delista (os medicamentos "raros essenciais"), cujos critérios deinclusão englobariam, entreoutros, a eficácia e a segurança desses medicamentos, sendo interrogada, entretanto, a necessidade de que existam estudos de custo-efetividade balizando essa inclusão ${ }^{34}$. 0 problema éque, como já mencionado, os medicamentos para doenças raras são aprovados para uso em seres humanos, pelos países desenvolvidos, de forma mais acelerada, na maioria das vezes sendo necessária apenas a realização de um ensaio clínico randomizado contra placebo com resultados positivos (geralmente com tamanho de amostra pequeno, e mostrando benefícios em desfechos não clínicos). Esta decisão éjustificada por vários fatores, entre os quaiso baixo número de pacientes existentes, e a fal ta de opções de tratamentos para essas doenças. Além disso, faltam estudos de história natural que permitam a realização de estudos de custo-efetividade (e, quando existentes, tais estudos costumam demonstrar que os medicamentos para doenças raras ultrapassam os limiares aceitáveis de custo-efetividade) ${ }^{9}$.

A inclusão de medicamentos para doenças raras em listas do MS poderia ser feita, na opinião dos autores, por meio de critérios diferenciados, utilizando princípios menos utilitaristas e levando em consideração tanto a vulnerabilidade da população atingida quanto a posição da sociedadeem relação a essa inclusão, com definição de prioridades.

\section{Aval iação detecnologias em saúde para doenças raras}

A medicina baseada em evidências ( $M B E$ ) nasceu na Universidade M acM aster, no Canadá, no início da década de 1980, e propõe que toda interven ção médica seja orientada pelas evidências obtidas a partir de experimentos científicos bem conduzidos ${ }^{35}$. Ela se apoia em quatro áreas distintas: epidemiologia clínica, metodologia científica, estatística e informática. A construção de recomendações ou diretrizes clínicas éuma parte importante da MBE, mas como existe uma produção contínua de informações, um aspecto fundamental éque devem existir estratégi as deatualização e de aprimoramento dessas diretrizes.

As diretrizes de tratamento são uma atividade preconizada pela Organização Mundial da 
Saúde. A produção de documentos baseados em evidências para orientação da conduta médica foi liderada, nos EUA e no Canadá, por iniciativas do governo junto às sociedades médicas e instituições acadêmicas a partir do final da década de $1970^{36}$. A primeira iniciativa do M S data de 2002, com a publicação dos "Protocolos Clínicos eD iretrizes Terapêuticas" (PCDT) para vários dos medicamentos excepcionais ${ }^{37}$. A maioria dos medicamentos para doenças genéticas incluídos na lista de medicamentos excepcionais apresenta PCDT publicado (Tabela 1).

A avaliação de tecnologias em saúde (ATS), por sua vez, é um processo que se inicia com a $M B E$, ou seja, com a busca e a análise de evidências sobre eficácia e segurança de determinada tecnologia, mas que leva em consideração, também, estudos econômicos e aspectos éticos e sociais para justificar alguma decisão em relação à sua inclusão (ou exclusão) em determinado programa/lista/rol de procedimento ${ }^{38,39}$. O s modelos atualmente existentes de ATS foram construídos para doenças prevalentes. No caso das doenças raras, as seguintes indagações continuam sem resposta adequada na literatura:

(1) As doenças raras devem ter algum "tratamento" diferenciado no tocante à ATS?

(2) É possível a realização de estudos de custo-efetividade para doenças raras?

(3) A política de incorporação de novas tecnologias para doenças raras deve ser pensada diferencialmente em países desenvolvidos e em desenvolvimento?

(4) Os métodos usuais de avaliação de ATS refletem as preferências da sociedade para o tratamento de doenças raras graves e/ou ameaçadoras da vida?

No Brasil, o governo regula o ciclo de vida das tecnologias em saúde através da Anvisa, do M Seda Agência Nacional de SaúdeSuplementar (ANS), todos locais privilegiados das atividades subsidiáveis pela ATS ${ }^{36}$. Decisões do Judiciário, entretanto, também influenciam a utilização de tecnologias de alto custo em nosso país $5^{36}$.

A Anvisa é o órgão responsável pelo registro das tecnologias e pelo monitoramento de sua utilização e efeitos adversos. 0 M S, por sua vez, está à frente de dois mecanismos regulatórios: 0 credenciamento para a provisão de procedimentos especiais ea definição de procedimentos cobertos pelo SUS. A ANS seria responsável pela delimitação dos procedimentos a serem cobertos pelas operadoras de planos de saúde ${ }^{36}$. Recentemente foi estruturada, também, a Comissão de Incorporação Tecnológica (Citec) do MS, que é a res- ponsável pela deliberação sobre solicitações de incorporações de tecnologias, análise de tecnologias em uso, revisão e mudanças de protocolos em saúdee de gestão do SU S e na Saúde Complementar ${ }^{40}$. Infelizmente, tais atores ainda não trabalham de forma integrada. Enquanto em alguns países, como nos EUA, a prescrição de medicamentos ainda não registrados pelo FDA pode ser o escopo de artigos publicados em revistas de impacto como o N ew England Journal of M edici$n \mathrm{e}^{41}$, no Brasil é plenamente aceitável que um medicamento seja prescrito sem que haja registro na Anvisa. Além disso, o registro de um medicamento não écondição única para sua inclusão em determinada lista de medicamentos no SUS.

A necessidade de uma política farmacêutica específica para doenças raras

Em facedo exposto, portanto, a existência deuma lista específica brasileira (e de uma política de assistência farmacêutica) para doenças raras justificar-se-ia, na opinião dos autores, pelos seguintes fatos:

- as doenças genéticas constituem um dos principais grupos de doen ças raras ${ }^{26}$ e são, cada vez mais, importante causa de mortalidade infantil no Brasil;

. $85 \%-90 \%$ das doenças raras são graves ou ameaçadoras da vida ${ }^{26}$;

- o número de pacientes diagnosticados com esse tipo de doença, embora ainda pequeno, tende a aumentar, seja pelo aumento da busca espontânea ou da busca de diagnóstico "financiada" pela indústria farmacêutica ou, ainda, pela maior acurácia dos testes diagnósticos;

- 0 custo dos medicamentos para essas doenças costuma ser muito elevado;

- a maioria dos tratamentos disponíveis para doenças genéticas não são fortemente baseados em evidências nem foram avaliados em relação a aspectos de custo-efetividade, em decorrência, entre outros aspectos, da falta de dados sobre história natural, de estímulo a esse tipo de pesquisa e de ensaios clínicos adequados;

- há falta de transparência em relação à inclusão/exclusão de medicamentos no CM DE, o que pode privilegiar certos grupos de doenças ou pacientes;

- existe variabilidade na conduta dos estados brasileiros em relação à implantação de programas para 0 atendimento dessas doenças;

- a maioria dos medicamentos para doenças raras, e que não estão incluídos em listas, são 
obtidos por meio de demandas judiciais. A necessidade de tratamento de muitos desses pacientes existe, e pode estar sendo postergada pela falta deuma política eficiente ou pelos gastos não justificados em medicamentos sem eficácia e segurança demonstrada.

\section{Conclusões}

A complexidade das opções terapêuticas medicamentosas atualmente disponíveis determina que as políticas de medicamentos não possam mais se restringir aos chamados itens essenciais e/ou de baixo custo. Esta nova realidade já levou, por exemplo, ao estabelecimento delistas dedicadas a medicamentos de maior custo dirigidos al gumas vezes a doenças raras. Junto a isto, em al guns casos, houve o estabelecimento de protocolos clínicos detratamento ediretrizes terapêuticas, cons- truídos para a racionalização do uso desses me dicamentos. A introdução de novas tecnologias de alto custo, em doenças raras, tem exemplos de sucesso dentre as doenças genéticas, sendo emblemático o caso da doença de Gaucher.

Uma das mais importantes questões é a de novas tecnologias que ainda não fazem parte da lista ou programa, mas que já se encontram no mercado e foram aprovadas por agências reguladoras; destas, muitas são indicadas para doenças raras, algumas das quais sem outras opções de tratamento. 0 maior desafio nessa área é ser capaz de estabelecer model os/processos de avaliação dessas tecnologias que agreguem aspectos técnicos eéticosequesejam capazes de distinguir o benefício terapêutico real desses medicamentos; isto éespecialmente prementequando consideramos a necessidade de que prioridades em saúde sejam definidas.

\section{Colaboradores}

MV Souza e IVD Schwartz foram responsáveis pela concepção, revisão da literatura e redação de todo o artigo; BC Krug e PD Picon participaram de parte da revisão da literatura e redação do artigo (com relação a políticas públicas e de medicamentos para doenças raras no Brasil). Todos os autores revisaram a versão final do artigo e concordaram com a sua submissão.

\section{Agradecimentos}

Os autores agradecem o apoio do M inistério da Ciência e Tecnologia(M CT)/Conselho Nacional de Desenvolvimento Científico e Tecnológico (CN Pq)/ M inistério da Saúde Secretaria deCiência, Tecnologia e Insumos Estratégicos-D epartamento de Ciência e Tecnologia (M S-SCTIE-DECIT) e a contribuição de Paulo Gilberto Cogo Leivas, Guilherme Becker Sander, Giácomo Balbinotto Netto e Raquel Tavares Boy da Silva em relação à discussão de aspectos específicos deste artigo. Agradecem, também, a Roberto Giugliani eCristina Brinckmann N etto pelos dados epidemiológicos fornecidos. 


\section{Referências}

1. Wraith JE. Lysosomal disorders. Semin Neonatol 2002; 7:75-83.

2. Meikle PJ, Hopwood JJ, Clague AE, Carey WF. Prevalence of lysosomal storage disorders. JAMA 1999; 281(3):249-254.

3. Poorthuis BJ, Wevers RA, Kleijer WJ, Groener JE, de Jong JG, van Weely $S$, Niezen-Koning KE, van Diggelen OP. The frequency of lysosomal storage diseases in The Netherlands. Hum Genet 1999; 105:151-156.

4. Coelho JC, Wajner M, Burin MG, Vargas CR, Giugliani R. Selective screening of 10.000 high-risk Brazilian patients for the detection of inborn errors of metabolism. Eur J Pediatr 1997; 156:650-654.

5. Brasil. M inistério da Saúde. Portaria no 3.916, de 30 de novembro de 1998. Aprova a Política Nacional de Medicamentos. Diário Oficial da União 1998; 10 nov.

6. Beck M. N ew therapeutic options for lysosomal storage disorders: enzyme replacement, small molecules and gene therapy. Hum Genet 2007; 121(1):1-22.

7. Picon $P$, Prolla $P, A$ maral $K$, Beutler $E$, Zimran $A$. Doença de Gaucher. In: Picon P, Beltrame A, organizadores. Protocolos clínicos e diretrizes terapêuticas. Brasília: M inistério da Saúde, 2002. p. 217-234.

8. Brasil. M inistério da Saúde. Pesquisa para a saúde. [site da Internet] [acessado $2007 \mathrm{dez}$ 15]. Disponível em: http://portal.saude.gov.br/portal/arquivos/ pdf/dirceubarbanodafsctiems.pdf

9. Connock M, Juarez-Garcia A, Frew E, M ans A, Dretzke J, Fry-Smith A, Moore D. A systematic review of the clinical effectiveness and cost-effectiveness of enzyme replacement therapies for Fabry's disease and mucopolysaccharidosis type 1 . Health Technol Assess 2006; 10(20):1-130.

10. Muñoz Rojas MV, Noratto DYJ. MPS I treatment option in Brazil, Latin America and worldwide: what does the MPS I registry tell us? Proceedings of the $10^{\text {th }}$ International Symposium on M ucopolysaccharide and Related Diseases. Vancouver, Canada; 2008 jun 26-29.

11. N eufeld E, Muenzer J. The mucopolysaccharidoses. In: Scriver CR, Beaudet AL, Sly WS, Valle $D$, editors. The metabolic and molecular basis of inherited disease. $7^{\text {th }}$ ed. New York: McGraw-Hill; 2001. p. 3421-3452.

12. Wraith JE, Clarke LA, Beck M, Kolodny EH, Pastores GM, M uenzer J, Rapoport DM, Berger KI, Swiedler SJ, Kakkis ED, Braakman T, Chadbourne E, WaltonBowen K, Cox GF. Enzyme replacement therapy for mucopolysaccharidosis I: a randomized, doubleblinded, placebo-controlled, multinational study of recombinant human $\alpha$-L-iduronidase (laronidase). J Pediatr 2004; 144(5):581-588.

13. The Canadian Expert Drug Advisory Committee (CEDAC). [site da Internet] [acessado 2008 jul 9]. Disponível em: acmts.ca/media/cdr/complete/ cdr_complete_Aldurazyme_2005July14.pdf

14. Muñoz Rojas $\bar{M} V$, Noratto $\bar{D} Y$ Jb. The MPS I registry: do patients in Brazil, Latin America and worldwide share the same phenotype distribution? Proceedings of the $10^{\text {th }}$ International Symposium on $\mathrm{M} \mathrm{u}$ copolysaccharide and Related Diseases. Vancouver, Canada; 2008 June 26-29.
15. Desnick RJ, Brady R, Barranger J, Collins AJ, Germain DP, Goldman M, Grabowski G, Packman S, Wilcox WR. Fabry disease, an under-recognized multisystemic disorder: expert recommendations for diagnosis, management, and enzyme replacement therapy. Ann Intern M ed 2003; 138(4):338-346.

16. $M$ acD ermot KD, Holmes $A$, M iners AH. AndersonFabry disease: clinical manifestations and impact of disease in a cohort of 98 hemizygous males. J M ed Genet 2001; 38(11):750-760.

17. Wraith JE. Clinical aspects and diagnosis. In: Platt FM, Walkley SU. Lysosomal disorders of the brain. Oxford: Oxford University Press; 2004. p. 50-80.

18. Eng CM, Guffon N, Wilcox WR, Germain DP, Lee P, Waldek S, Caplan L, Linthorst GE, Desnick RJ. Safety and efficacy of recombinant human alphagalactosidase A-replacement therapy in Fabry's disease. N Engl J M ed 2001; 345(1):9-16.

19. Banikazemi $M$, Bultas J, Waldek $S$, Wilcox WR, Whitley $C B, M$ CD onald $M$, Finkel R, Packman $S$, Bichet DG, Warnock DG, Desnick RJ. Agalsidasebeta for advanced fabry disease: a randomized trial. Ann Int M ed 2007; 146:77-86.

20. Schiffmann R, Kopp JB, Austin HA, III, Sabnis S, Moore DF, Weibel T, Balow JE, Brady RO. Enzyme replacement therapy in Fabry disease: a randomized controlled trial. JAM A 2001; 285(21):2743-2749.

21. Vedder AC, Linthorst GE, Houge G, Groener JE, Ormel EE, Bouma BJ, Aerts JM, Hirth A, Hollak CE. Treatment of Fabry disease: outcome of a comparative trial with agalsidase alfa or beta at a dose of 0.2 $\mathrm{mg} / \mathrm{kg}$. PLoS One 2007; 2(7):e598.

22. The Canadian Expert Drug Advisory Committee (CEDAC). [site da Internet] [acessado 2008 jul 9]. Disponível em: cadth.ca/media/cdr/complete/ cdr_complete_Replagal_2004N ov24.pdf

23. The Canadian Expert Drug Advisory Committee (CEDAC). [site da Internet] [acessado 2008 jul 9]. Disponível em: www.acmts.ca/media/cdr/complete/ cdr_complete_Fabrazyme_Resubmission_may2005.pdf

24. Health Canada. [site dä Internet] [acessado 2007 dez 15]. Disponível em: http://www.hc-sc.gc.ca/ ahc-asc/media/nr-cp/2006/2006_48_e.html

25. Silva R. M edicamentos excepcionais no âmbito da assistência farmacêutica no Brasil [dissertação]. Rio de Janeiro: Escola Nacional de Saúde Pública Sergio Arouca, Fundação Oswaldo Cruz; 2000.

26. Haffner ME. Focus on research: adopting orphan drugs - two dozen years of treating rare diseases. $\mathrm{N}$ Engl J M ed 2006; 354(5):445-447.

27. USA. Food and Drug Administration (FDA). [site da Internet] [acessado $2007 \mathrm{dez}$ 15]. Disponível em: http://www.fda.gov/orphan/index.htm

28. European M edicines Agency (EMEA). [site da Internet] [acessado 2007 set 15]. Disponível em: http:// www.emea.eu.int/htms/human/comp/orphapp.htm

29. Brasil. Ministério da Saúde. Agência Nacional de Vigilância Sanitária. Glossário da Anvisa. [site da Internet] [acessado $2007 \mathrm{dez}$ 15]. Disponível em: http://e-glossario.bvs.br/glossary/public/scripts/ php/page_search.php?lang $=\&$ letter $=M$ ) 
30. Brasil. M inistério da Saúde. Portaria GM/M S no 2.577, de 27 de outubro de 2006. Aprova o componente de medicamentos de dispensação excepcional. Diário Oficial da União 2006; 10 nov.

31. Souza R. O Programa de M edicamentos Excepcionais. In: Picon P, Beltrame A. Protocolos clínicos e diretrizes terapêuticas. Brasília: Ministério da Saúde; 2002.

32. Picon DP, Beltrame A, organizadores. Ministério da Saúde: protocolos clínicos e diretrizes terapêuticas medicamentos excepcionais. v. 1. Porto Alegre: Gráfica Pallotti; 2002.

33. Krug B, Schwartz I. Doença de Gaucher: delineando estratégias para promoção do uso racional de imiglucerase no Brasil. Anais do XVIII Congresso Brasileiro de Genética Clínica; 2006. p. 58-59.

34. Stolk P, Willemen MJC, Leufkens HGM . "Rare essentials": drugs for rare diseases as essential medicines. Bulletin of the World Health Organization 2006; 84(9):745-751.

35. Evidence-Based Medicine Working Group. Evidence-based medicine: a new approach to teaching the practice of medicine. JAM A 1992; 268:2420-2425.

36. Silva L. Avaliação tecnológica e análise custo-efetividade em saúde: a incorporação de tecnologias e a produção de diretrizes clínicas para o SUS. Cien Saude Colet 2003; 8(2):501-520.

37. Brasil. Ministério da Saúde. Secretaria de Assistência à Saúde. Departamento de Sistemas e Redes Assistenciais. Protocolos clínicos e diretrizes terapêuticas: medicamentos excepcionais. Série A: normas e manuais técnicos. Brasília: M inistério da Saúde; 2002.

38. Novaes HMD. Avaliação de programas, serviços e tecnologias em saúde. Rev Saude Publica 2000; 34(5):547-559.

39. Williams AH, Cookson RA. Equity-efficiency tradeoffs in health technology assessment. International Journal of Health Technology Assessment 2006; 22(1):1-10

40. Brasil. M inistério da Saúde. Portaria GM /M S no 3.223, de 27 de dezembro de 2006. Institui a comissão para incorporação de tecnologias no âmbito do Sistema Ú nico de Saúde e da Saúde Suplementar. Diário Oficial da União 2006; 28 dez.

41. Okie S. Acces before approval: a right to take experimental drugs? N Engl J M ed 2006; 355(5):437-440.

42. Cogan J, Phillips J. Inherited defects in growth hormone synthesis and action. In: Scriver CR, Beaudet $A L$, Sly WS, Valle D, editors. The metabolic and molecular basis of inherited disease. $7^{\text {th }}$ ed. New York: M cGraw-Hill; 2001. p. 4159-4180.

43. Culotta V, Gitlin J. Disorders of Copper Transport. In: Scriver CR, Beaudet AL, Sly WS, Valle D, editors. The metabolic and molecular basis of inherited disease. $7^{\text {th }}$ ed. New York: McGraw-Hill; 2001. p. 3105-3126.
44. First $\mathrm{H}, \mathrm{H}$ urt J, editors. Oxford desk reference clinical genetics. Oxford: Oxford University Press, Southern Africa; 2005.

45. Brasil. Ministério da Saúde. Protocolos clínicos e diretrizes terapêuticas: medicamentos excepcionais; 2006. [site da Internet] [acessado 2008 jul 8]. Disponível em: http://portal.saude.gov.br/saude/ visualizar_texto.cfm?idtxt $=25262$

46. Brasil. M inistério da Saúde. Protocolos clínicos e diretrizes terapêuticas: medicamentos excepcionais; 2002. [site da Internet] [acessado 2008 jul 8]. Disponível em: http://dtr2001.saude.gov.br/sas/dsra/ protocolos/index.htm

47. Brasil. Ministério da Saúde. Consulta pública no 3 , de 13/06/2008. Protocolo clínico e diretrizes terapêuticas: angioedema hereditário. [site da Internet] [acessado 2008 jul 8]. Disponível em: http:// dtr2001.saúde.gov.br/sas/PORTARIAS/Port2008/ PT-03-CONS.htm

48. Brasil. M inistério da Saúde. Protocolos clínicos e diretrizes terapêuticas: medicamentos excepcionais; 2007. [site da Internet] [acessado 2008 jul 8]. Disponível em: http://portal.saude.gov.br/portal/saude/visualizar texto.cfm?idtxt=27836

49. Brasil. Ministério da Saúde. Portaria no 2.305, de 19 de dezembro de 2001. Aprova o Protocolo de Indicação de Tratamento Clínico da osteogenesis imperfecta com pamidronato dissódico no âmbito do Sistema Ú nico de Saúde (SUS). Diário O ficial da U nião 2001; 20 dez.

50. Wraith JE. Limitations of enzyme replacement therapy: current and future. J Inherit M etab Dis 2006; 29(2-3):442-447.

51. Muenzer J, Wraith JE, Beck M, Giugliani R, Harmatz $P$, Eng CM, Vellodi A, M artin R, Ramaswami U, Gucsavas-Calikoglu M, Vijayaraghavan S, Wendt S, Puga AC, Ulbrich B, Shinawi M, Cleary M, Piper D, Conway AM, Kimura A. A phase II/III clinical study of enzyme replacement therapy with idursulfase in mucopolysaccharidosis II (Hunter syndrome). Genet M ed 2006; 8(8):465-473.

52. Clinical trials. [site da Internet] [acessado $2007 \mathrm{dez}$ 15]. Disponível em: http://www.clinicaltrials.gov

Artigo apresentado em 10/11/2007

Aprovado em 10/06/2008

Versão final apresentada em 06/08/2008 